\title{
Using Design Patterns for Math Preservice Teacher Education
}

\author{
Paul Salvador Inventado \\ California State University Fullerton \\ Fullerton, California \\ pinventado@fullerton.edu \\ Yeping Li \\ Texas A\&M University \\ College Station, Texas \\ yepingli@tamu.edu \\ Neil Heffernan \\ Worcester Polytechnic Institute \\ Worcester, Massachusetts \\ nth@wpi.edu
}

\author{
Sharris Gayle Francisco \\ Inventado \\ Carnegie Mellon University \\ Pittsburgh, Pennsylvania \\ sfrancis@andrew.cmu.edu
}

Peter Scupelli
Carnegie Mellon University
Pittsburgh, Pennsylvania
pgs@andrew.cmu.edu
Shihfen Tu
University of Maine
Orono, Maine
shihfen.tu@maine.edu

\author{
Noboru Matsuda \\ Texas A\&M University \\ College Station, Texas \\ noboru.matsuda@tamu.edu
}

\author{
Korinn Ostrow \\ Worcester Polytechnic Institute \\ Worcester, Massachusetts \\ ksostrow@wpi.edu \\ Craig Mason \\ University of Maine \\ Orono, Maine \\ craig.mason@maine.edu
}

\author{
Mary Logue \\ University of Maine \\ Orono, Maine \\ mary.logue@maine.edu
}

\author{
Pat McGuire \\ University of Colorado Colorado \\ Springs \\ Colorado Springs, Colorado \\ pmcguire@uccs.edu
}

\begin{abstract}
Experienced teachers effectively address student misconceptions and learning difficulties by employing high-quality pedagogical strategies that they have developed through experience. It is difficult to discover effective strategies because it takes a lot of trial and error before a teacher can conclude that a particular approach improves student learning. Researchers have used educational design patterns to encapsulate high-quality strategies that facilitate the transfer of pedagogical knowledge. In this work, we investigated the feasibility of utilizing three educational design patterns as a teaching tool for math preservice teacher education: Feedback Sandwich, Pitfall Diagnosis and Prevention, and Worked Examples. Specifically, we used design patterns to investigate how preservice teachers provided feedback to students who gave common wrong answers to a given math problem and compared their feedback before and after they were introduced to educational design patterns. Results indicated that design patterns helped preservice teachers consider other aspects of feedback such as students' self-regard, common misconceptions, and prior knowledge into their feedback for common wrong answers on math problems. The limited set of three design patterns introduced in the study focused on feedback

Permission to make digital or hard copies of all or part of this work for personal or classroom use is granted without fee provided that copies are not made or distributed for profit or commercial advantage and that copies bear this notice and the full citation on the first page. Copyrights for components of this work owned by others than the author(s) must be honored. Abstracting with credit is permitted. To copy otherwise, or republish, to post on servers or to redistribute to lists, requires prior specific permission and/or a fee. Request permissions from permissions@acm.org.

EuroPLoP '18, fuly 4-8, 2018, Irsee, Germany

(c) 2018 Copyright held by the owner/author(s). Publication rights licensed to ACM. ACM ISBN 978-1-4503-6387-7/18/07 ..\$15.00

https://doi.org/10.1145/3282308.3282340
\end{abstract}

presentation. Results also indicated preservice teachers may benefit from design patterns that address other aspects of feedback such as content granularity, feedback length, and presentation medium. Implications of this study, including the potential of using educational design patterns to improve preservice teacher education, are discussed.

\section{CCS CONCEPTS}

- Software and its engineering $\rightarrow$ Design patterns; $\bullet$ Applied computing $\rightarrow$ E-learning;

\section{KEYWORDS}

preservice teacher education, educational design patterns, evaluation, ASSISTments

\section{ACM Reference Format:}

Paul Salvador Inventado, Sharris Gayle Francisco Inventado, Noboru Matsuda, Yeping Li, Peter Scupelli, Korinn Ostrow, Neil Heffernan, Shihfen Tu, Craig Mason, Mary Logue, and Pat McGuire. 2018. Using Design Patterns for Math Preservice Teacher Education. In 23rd European Conference on Pattern Languages of Programs (EuroPLoP '18), July 4-8, 2018, Irsee, Germany. ACM, New York, NY, USA, 8 pages. https://doi.org/10.1145/3282308.3282340

\section{INTRODUCTION}

Many of the best teachers in the world are effective because of the amount of time and effort they put into determining and testing pedagogical strategies that impact student learning. Educational researchers often work with such experienced teachers to find high-quality pedagogical strategies in an attempt to replicate their effectiveness in diverse student populations and varied educational contexts. One of the challenges, however, is not only expanding 
Paul Salvador Inventado, Sharris Gayle Francisco Inventado, Noboru Matsuda, Yeping Li, Peter Scupelli, Korinn Ostrow, Neil Heffernan, EuroPLoP '18, July 4-8, 2018, Irsee, Germany

Shihfen Tu, Craig Mason, Mary Logue, and Pat McGuire

pedagogical knowledge but also passing it down to the next generation of teachers so they may leverage well-researched and effective strategies. In this paper, we focus on how pedagogical knowledge can be introduced to preservice teachers specifically within the context of using design patterns to give feedback.

The term preservice teacher is used to refer to college students who are being trained to become teachers. During their time in teacher preparation programs, preservice teachers often focus on furthering their content knowledge, learning how to align their teaching to state and national standards, and learning how to assess student learning. The curriculum and standards mandate what preservice teachers must learn but these inexperienced teachers will soon find themselves in classrooms where they need to know and decide how to assess student learning accurately and effectively.

One skill preservice teachers need to learn, for example, is the ability to remediate errors in classrooms as needed in effective instruction. This skill is an underlying concept in Gudmundsdottir and Shulman's 1987 pedagogical content knowledge that is essential for effective teaching. The teachers must understand the content, understand students' thinking that contributes to incorrect answers, and understand how to give meaningful feedback to remediate students' underlying misconceptions. These layers of skill and knowledge, evident in many experienced teachers must be learned by preservice teachers within a limited time. In many cases, preservice teachers are provided opportunities to engage in practice teaching (i.e., student teaching), but often these experiences do not cover scenarios they may face when they begin their teaching career, particularly those related to remediation of students' common errors.

Researchers and practitioners document pedagogical knowledge in many ways such as evidence-based practices, design principles, and, recently, design patterns that are potentially useful for preparing preservice teachers. Compared to design principles, educational design patterns describe high-quality solutions to recurring problems in a specific educational context [10]. The structure of design patterns facilitates their application because teachers only need to check whether a learning scenario matches a particular problem and educational context to identify an appropriate solution. Design patterns keep the novice teacher's focus on how to teach in ways that address misunderstanding.

This work attempts to answer the question of whether educational design patterns can facilitate the transfer of pedagogical knowledge in math preservice teacher education. We conducted a study that compared feedback created by preservice teachers before and after they were introduced to three educational design patterns. Results offer insights on how well preservice teachers understood the pedagogical knowledge encapsulated by design patterns and how much it influenced the feedback they constructed for their potential students.

\section{REVIEW OF RELATED LITERATURE}

Design patterns were first introduced by Christopher Alexander to the field of Architecture in the late 1970's [1]. In this work, Alexander and colleagues developed an architectural pattern language that consisted of design patterns to explain why certain commonly-observed situations caused problems and to develop routine solutions. For example, the Pedestrian Street design pattern increased social interaction while keeping people safe from traffic by providing a space between buildings and the street.

Design patterns have been adapted in various disciplines. In Software Engineering, they are used to encapsulate reusable software designs that effectively address common issues like scalability, robustness, and security (see for example [5]). In the field of Humancomputer Interaction, design patterns are used to capture interfaces that successfully support user interactions in computer-based environments such as navigation, task-support, and conveying information (see for example [17]). Management design patterns embody best practices that address management challenges such as communication, collaboration, and improvement (see for example [14]). Education design patterns describe high-quality teaching and learning strategies that address barriers to learning such as motivation, appropriate feedback, and inclusive learning environments (see for example [3]).

Several authors have developed educational design patterns to capture high quality solutions for recurring problems in different learning contexts such as class lectures and activities [3], flipped classrooms [13], MOOCs [19], active learning [8], and creative thinking [12].

Inventado et al. 2016 conducted a study to evaluate the effectiveness of design patterns on student learning using a data-driven approach. Their analysis of the Hint on Demand design pattern [20] confirmed the effectiveness of on-demand hints for math homework given to students during the regular academic year through an online learning system. However, they also found that on-demand hints were less effective in a replication experiment conducted on a different student population who answered math problems over the summer as preparation for the next school year. Results indicated the importance of selecting appropriate design patterns that fit the learning context to support student learning.

Design patterns have also been used as boundary objects to facilitate discussions among participants with diverse backgrounds [15]. Studies show the effectiveness of using design patterns to facilitate communication in various domains including those with multidisciplinary teams like education [16], design [4], and software development [18].

\section{METHODOLOGY}

The goal of this study was to investigate the feasibility of introducing design patterns as a tool for helping preservice teachers give remedial feedback to students. Specifically, we asked preservice teachers to construct feedback for students who submit common wrong answers to a specific math problem. We compared the feedback preservice teachers constructed before and after they were introduced to design patterns. We expected preservice teachers to successfully create feedback based on the design patterns they learned and their feedback to address critical issues they had not previously considered such as students' self-regard, common misconceptions, and lack of prior knowledge.

\subsection{Identifying Common Wrong Answers}

ASSISTments is an online learning system created in 2002 that is now used by more than 50,000 students around the United States 


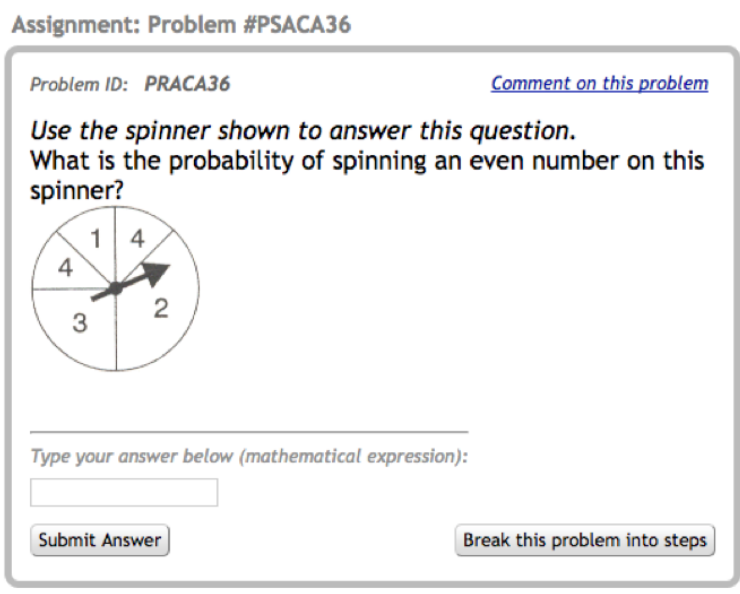

Figure 1: Spinner problem (Screenshot courtesy of www.assistments.org)

[7]. The system and its content were developed by a team of computer scientists, learning scientists, and teachers. The majority of ASSISTments users are middle-school students. Most of the system's content is math-related, but it also contains problems for other domains like English, Chemistry, and Biology. Teachers often use the system to assign math exercises to their students. The system automatically assesses students' responses and provides them helpful feedback even when a teacher is unavailable. Such help also reduces teachers' workload and gives them more time to perform other teaching tasks. The system generates reports about student performance that teachers can use to adapt their teaching strategies to better address the needs of their students. These reports provide teachers with powerful information on common wrong answers, allowing them to address specific areas of struggle within an assignment.

The present work leveraged this common wrong answer data to design case studies for preservice teachers to work with while using design patterns. These case studies provided preservice teachers with examples of real student misconceptions and a unique opportunity to analyze student work and provide appropriate feedback. Figures 1 and 2 provide screenshots of the problems we selected, referred to in this paper as the Spinner problem and the Venn diagram problem. Both problems were selected because historical data showed that these problems were answered by many students (i.e., over 1,000) and that students frequently submitted a common wrong answer (i.e., more than $40 \%$ of all incorrect answers were of a certain form). Table 1 provides more specific details about each problem and its common wrong answer.

\subsection{Design Patterns}

We explored the effectiveness of using design patterns in preservice teacher education by introducing three design patterns that addressed common challenges in providing feedback: namely, Feedback Sandwich [3], Pitfall Diagnosis and Prevention [2], and Worked Examples [9]. We chose these patterns because they guide the creation of feedback content that preservice teachers

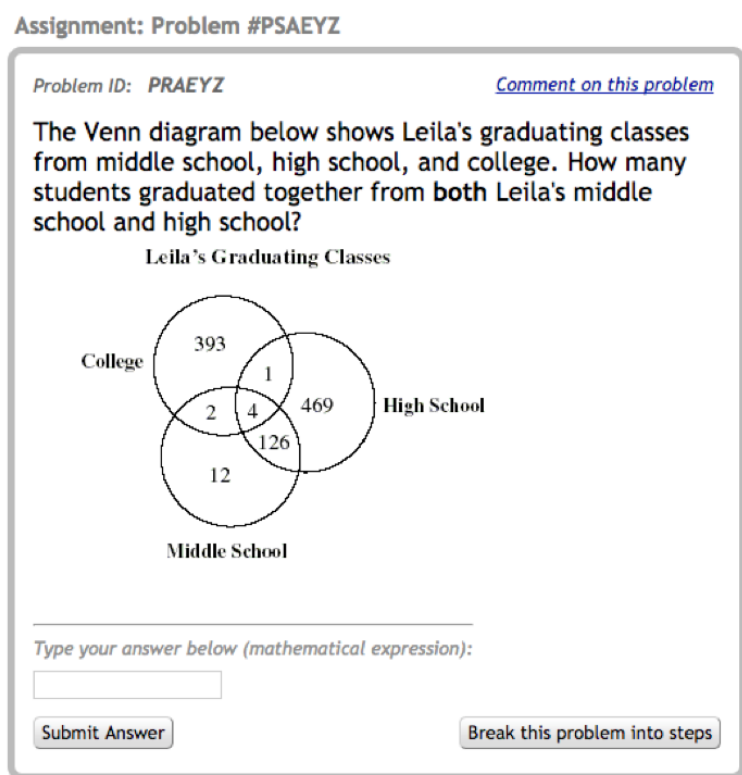

Figure 2: Venn diagram problem (Screenshot courtesy of www.assistments.org)

need to remediate students' misconceptions. Table 2 presents a summary of these three design patterns.

Feedback Sandwich is used when students struggle with a learning activity and experience negative emotions that may cause them to give up. The positive feedback aims to reassure student efforts, encourage them to be receptive to suggestions, and motivate them to persevere. We expected preservice teachers to easily understand and implement this design pattern because it is straightforward and it produces relatively short instances of feedback.

Pitfall Diagnosis and Prevention is used when teachers notice that students give common wrong answers to a problem. This indicates a common misconception so it helps to provide suggestions that help students to revise this misconception and avoid it in the future. We anticipated preservice teachers would find it easy to understand the design pattern, but would struggle with implementation due to their lack of teaching experience. It is difficult to spot common wrong answers unless you have seen many student solutions to a problem. Similarly, it is difficult to identify effective suggestions unless you have some background knowledge about the problem. For example, it is easier to give feedback when you have experienced solving a problem and received suggestions that you found effective, learned strategies from experienced teachers, or know effective strategies for a similar problem.

Worked Examples are used when students forget or have misconceptions about the process used to solve a problem. Solutions to similar problems help students understand the concept and provide a guide they can use to solve the current problem. We suspected preservice teachers would find it easy to understand this design pattern, but similar to the Pitfall Diagnosis and Prevention design pattern, may struggle with its implementation. On the surface level, the Worked Example design pattern seems simple because it only requires a demonstration for solving a similar problem, but 
Paul Salvador Inventado, Sharris Gayle Francisco Inventado, Noboru Matsuda, Yeping Li, Peter Scupelli, Korinn Ostrow, Neil Heffernan, EuroPLoP '18, July 4-8, 2018, Irsee, Germany Shihfen Tu, Craig Mason, Mary Logue, and Pat McGuire

Table 1: Historical data collected on selected ASSISTments problems

\begin{tabular}{lcccc}
\hline Problem & Student Solutions & Correct Answer & Common Wrong Answer & Common Wrong Answer Frequency \\
\hline Spinner & 1,054 & $5 / 8$ & $3 / 5$ & $41 \%$ \\
Venn diagram & 4,781 & 130 & 126 & $58 \%$ \\
\hline
\end{tabular}

Table 2: Summary of design patterns used in the study

\begin{tabular}{ll}
\hline Design Pattern & Summary \\
\hline Feedback Sandwich [3] & $\begin{array}{l}\text { In giving feedback, start and end with positive feedback with any criticism in } \\
\text { between in order to maximize student self-regard. Suggestions for improvement } \\
\text { are sandwiched between these reinforcing comments. }\end{array}$ \\
Pitfall Diagnosis and Prevention [2] & $\begin{array}{l}\text { Pay special attention to vital concepts and emphasize them when it has shown } \\
\text { that last time you taught the concept students had trouble with it. } \\
\text { Provide students with an example similar to the problem they are asked to solve } \\
\text { so they understand how to solve the problem without revealing the answer. }\end{array}$ \\
\hline
\end{tabular}

in reality it requires a strong understanding of the concept and and underlying ability to identify similar problems, and provide correct and clear explanations. In addition, Worked Examples often require more effort and detail, particularly when the solution involves many steps.

\section{USING DESIGN PATTERNS FOR FEEDBACK CONSTRUCTION}

We investigated the effectiveness of design patterns for preservice math education by conducting a study on a mandatory preservice education course at a tier-1 public research university for undergraduate students in the United States. Specifically, design patterns were introduced in one of the class' discussions about providing feedback to students' common wrong answers in math problems.

The class considered in the study consisted of 32 undergraduate preservice teachers and was offered in the Fall 2015 semester. Out of the 32 preservice teachers, 21 were sophomores, 10 were juniors, and one was a senior. Twenty-seven preservice teachers intended to teach mathematics following their studies, while five of them indicated that they were not likely to end up teaching math. Of these five preservice teachers, four anticipated that they would be middle school teachers, while one anticipated becoming a high school teacher teaching a subject other than math.

The study introduced one activity per week in four consecutive classroom sessions. In the first part of the study, the preservice teachers were introduced to the ASSISTments online learning system to better understand how the system worked and the type of data it collected. They were given a homework assignment that asked them to solve the Spinner problem, identify possible misconceptions that led to the common wrong answer, and write feedback that they thought would help address students' misconceptions about the problem. Their answers were collected using ASSISTments to facilitate data collection. We refer to this as Task 1: Naturalistic text feedback.

After preservice teachers submitted their homework assignment, they listened to a 30-minute lecture on educational design patterns.
The lecture provided a short history of design patterns, the format and usage of design patterns, and descriptions of our three specific design patterns of interest: Feedback Sandwich, Pitfall Diagnosis and Prevention, and Worked Examples.

Preservice teachers were then given another homework assignment that asked them to answer the Venn diagram problem, identify possible misconceptions that led to the common wrong answer, and provide feedback for students who submitted the common wrong answer. In order to facilitate their use of design patterns, preservice teachers were asked to select one of the three design patterns discussed in the lecture and write feedback using the design pattern. We refer to this task as Task 2: Design-pattern-based text feedback. Preservice teachers were then asked to select a design pattern and create a video of themselves giving feedback to their future students. Preservice teachers were allowed to use the same design pattern from the first task or use a different design pattern. We refer to this task as Task 3: design-pattern-based video feedback.

Both homework assignments were graded by the instructor and the data collected from ASSISTments were used for analyses.

\section{RESULTS}

The data collected from each feedback creation task were analyzed separately and compared with results from other feedback creation tasks. Preservice teachers' answers were manually analyzed to uncover commonly used feedback features that are described in Table 3. Table 4 provides their preferences for selecting design patterns in each task. Table 5 summarizes the percentage of student solutions that exhibited a feedback feature in each task.

\subsection{Task 1: Naturalistic text feedback}

The data collected from Task 1 revealed that the majority of preservice teachers' naturalistic feedback focused on the content. As Table 5 shows, most preservice teacher solutions exhibited detailed explanations (84\%) as well as procedural (81\%) and conceptual (88\%) explanations. Interestingly, half of the preservice teachers' feedback explicitly informed students of their answers' incorrectness. The 
Table 3: Commonly-used features in preservice teacher feedback

\begin{tabular}{ll}
\hline Feedback features & Description \\
\hline Detailed Explanation & $\begin{array}{l}\text { Thorough explanation for solving the problem that often used examples and/or } \\
\text { visualization }\end{array}$ \\
Rejection & Explicitly states that the student's answer was incorrect \\
Compliment & Praise student effort \\
Review & Brief reminder of a previous lesson or topic \\
Students' POV & Recognize reasons that might have led the student to provide the incorrect answer \\
Brief Explanation & Concise explanation for solving the problem (e.g., absent of examples, procedures, \\
& and/or visualization) \\
Suggestion & Provide recommendations to help solve the problem \\
Reassurance & Affirmation of students' capabilities \\
Encouragement & State that students' perseverance will be rewarded with success \\
In-Depth Look at Question/ Problem & Highlight important aspects of the problem that may help students identify the \\
& correct answer or identify misconceptions \\
Procedural Justification & Provide mathematical procedure required in solving the problem \\
Conceptual Justification & Explain mathematical concepts required in solving the problem \\
Correct answer & Provide correct answer \\
Incorrect answer & Provide incorrect answer \\
\hline
\end{tabular}

Table 4: Preservice teacher preferences in selecting design patterns for each task ${ }^{*}$

\begin{tabular}{lrr}
\hline Design pattern & Task 2 $(N=32)$ & Task 3 $(N=12)$ \\
\hline Feedback Sandwich & $81.25 \%$ & $48.39 \%$ \\
Pitfall Diagnosis and Prevention & $15.63 \%$ & $35.48 \%$ \\
Worked Examples & $3.12 \%$ & $16.13 \%$ \\
\hline
\end{tabular}

${ }^{*}$ Note: Task 2 and Task 3 asked for preservice teacher feedback after introducing them to design patterns. Task 2 asked for text feedback, while Task 3 asked for video feedback.

Table 5: Percentage of student solutions that exhibited a feedback feature in each task.*

\begin{tabular}{lrrr}
\hline Feedback features & Task $1(N=32)$ & Task $2(N=32)$ & Task 3 $(N=12)$ \\
\hline Detailed Explanation & $\mathbf{8 4 . 3 8} \%$ & $54.55 \%$ & $41.67 \%$ \\
Rejection & $\mathbf{5 0 . 0 0} \%$ & $3.03 \%$ & $0.00 \%$ \\
Compliment & $25.00 \%$ & $\mathbf{4 8 . 4 8} \%$ & $33.33 \%$ \\
Review/Reminder & $\mathbf{2 1 . 8 8} \%$ & $18.18 \%$ & $16.67 \%$ \\
Students' POV & $12.50 \%$ & $\mathbf{5 1 . 5 2} \%$ & $50.00 \%$ \\
Brief Explanation & $12.50 \%$ & $42.42 \%$ & $\mathbf{5 0 . 0 0} \%$ \\
Suggestion & $15.63 \%$ & $21.21 \%$ & $\mathbf{2 5 . 0 0} \%$ \\
Reassurance & $3.13 \%$ & $69.70 \%$ & $\mathbf{7 5 . 0 0} \%$ \\
Encouragement & $3.13 \%$ & $\mathbf{6 6 . 6 7} \%$ & $58.33 \%$ \\
In-Depth Look at Question/ Problem & $3.13 \%$ & $\mathbf{7 5 . 7 6} \%$ & $50.00 \%$ \\
Procedural Justification & $\mathbf{8 1 . 2 5} \%$ & $51.52 \%$ & $41.67 \%$ \\
Conceptual Justification & $87.50 \%$ & $\mathbf{9 6 . 9 7 \%}$ & $91.67 \%$ \\
Correct answer & $81.25 \%$ & $81.82 \%$ & $\mathbf{9 1 . 6 7 \%}$ \\
Incorrect answer & $12.50 \%$ & $\mathbf{1 5 . 1 5} \%$ & $8.33 \%$ \\
\hline
\end{tabular}

*Note: Task 1 asked preservice teachers to provide naturalistic text feedback, while Task 2 and Task 3 asked for feedback after introducing them to design patterns. Task 2 asked for text feedback, while Task 3 asked for video feedback. Numbers in bold indicate the task where the feedback feature was most prevalent in student solutions. 
Paul Salvador Inventado, Sharris Gayle Francisco Inventado, Noboru Matsuda, Yeping Li, Peter Scupelli, Korinn Ostrow, Neil Heffernan, EuroPLoP '18, July 4-8, 2018, Irsee, Germany Shihfen Tu, Craig Mason, Mary Logue, and Pat McGuire

feedback below shows one preservice teacher's feedback for the Spinner problem that illustrates these three features.

The answer 3/5 is wrong because there are eight total wedges. Because there are eight total wedges in the spinner, the answer is 5/8. To find the correct answer, we must look at the smallest wedges and continue counting them around the circle, even in the bigger sections. So, we count that there are two smaller wedges in the number 3 wedge, and there are three smaller wedges in the number 2 wedge, which makes eight total wedges. We then must count how many even number wedges there are, which, after adding in the visuals of the two wedges in wedge 3 and the three wedges in wedge 2, we can see that there are 5 wedges with even numbers (two \#4 wedges, and three \#2 wedges). When we find probability, we take the number of the outcome which is 5, and put it over the total number of possible outcomes, which is 8. So, the answer is $5 / 8$.

\subsection{Task 2: Text feedback after the introduction to design patterns}

The data collected from Task 2 showed that the majority of the preservice teachers selected the Feedback Sandwich design pattern $(81 \%)$. The pattern's goal is to maximize students' self-regard which can explain why most feedback collected contained text that took the students' point of view (52\%), complimented students' effort (49\%), provided reassurance (70\%) and gave encouragement $(67 \%)$. Take note that there was hardly any feedback that called out students' wrong answers (3\%). Most feedback focused on math concepts (97\%) and highlighted specific misconceptions in the problem $(76 \%)$ rather than talking about specific procedures (51\%). Perhaps preservice teachers felt that it was better to focus on specific issues rather than overwhelm students with too much information. The feedback below shows one preservice teacher's feedback for the Venn diagram problem that illustrates the previously mentioned features.

Good job in identifying the part of the Venn diagram that holds BOTH the middle and high school graduates! However, the number in the very middle of the Venn diagram, 4 , needs to be added to 126, because the students that graduated from middle school, high school, and college also graduated from middle and high school. You did an excellent job on identifying what the problem was asking.

\subsection{Task 3: Video feedback after the introduction to design patterns}

There were only 12 out of 32 video feedback clips available for analysis due to data sharing and accessibility issues. Some participants did not grant us access to their videos for research. However, the available data showed that almost half of the preservice teachers selected the Feedback Sandwich design pattern and most of the others selected the Pitfall Diagnosis and Prevention design pattern (33\%). On average, the video feedback duration was $48 \mathrm{sec}-$ onds, which could explain why there were fewer occurrences of detailed feedback. Instead, feedback seemed straightforward that provided the correct answer (92\%) and focused on conceptual explanations (92\%). Interestingly, although the Pitfall Diagnosis and Prevention design pattern does not specifically focus on student's self-regard, most of the feedback provided reassurance (75\%), which is a feature closely associated with the Feedback Sandwich design pattern. The transcript below was taken from a preservice teacher's video feedback for the Venn diagram problem that illustrates the previously mentioned features.

It is easy to get confused by Venn diagram be-
cause what the problem is asking for can fall un-
der different sections of the Venn diagrams. But I
very much understand why you had issues with
this problem today. Umm this problem is tricky
because not only are we looking âAe we're look-
ing for Leila's class that graduated umm from
her middle school and her high school so this
falls into two different sections. That falls into
the section that you recognized where she gradu-
ated from her middle school high school but also
falls underneath the section where she graduated
from her middle school high school and elemen-
tary school. So this must be kept in mind. I'm
sure in the future as long as you read the prob-
lem very carefully and understand what they're
asking for you'll able ... be able to decipher the
Venn diagram and find the correct answer in the
future.

\section{DISCUSSION}

The results showed a noticeable change in preservice teacher feedback indicating that they understood the design patterns and were able to apply them. In Task 2, for example, majority of the preservice teachers selected the Feedback Sandwich design pattern. Compared to Task 1, preservice teachers created feedback that aligned with the pattern such that they gave more attention to students' self-regard by avoiding statements that explicitly rejected students' answers, complimenting student effort, understanding why students made mistakes, reassuring students' capabilities, and encouraging them to keep trying.

Pitfall Diagnosis and Prevention was the second most preferred design pattern for Task 2 and Task 3. Preservice teachers did not need as much prior teaching experience to apply the pattern because they were given common wrong answers to ASSISTments problems. However, preservice teachers still needed to analyze the data to uncover underlying misconceptions and identify feedback to address them. Preservice teachers who selected this pattern correctly applied its definition such that their feedback highlighted student misconceptions, discussed concepts that addressed specific errors, and gave suggestions to avoid the incorrect solutions.

Preservice teachers were instructed to construct feedback for students who submitted a common wrong answer without specifying particular learning scenarios. As a result, they could freely 
imagine learning contexts and apply design patterns they felt would properly address it. We think that some of the driving forces behind the preservice teachers' decisions included the prevalence of the learning context, the difficulty of implementing the solution, and the simplicity of the design pattern.

Most preservice teachers selected the Feedback Sandwich design pattern and successfully constructed feedback for students struggling with a misconception. We did not ask the preservice teachers about why they chose this design pattern, but hypothesized that it was because the pattern addresses a common learning context, the solution is straightforward, and the pattern is simple. Specifically, it is common for students to struggle with a math problem and lose confidence and motivation to answer it. The solution is quite easy to implement as it only involves providing encouraging statements and feedback that addresses a particular misconception.

We hypothesized that preservice teachers preferred the Pitfall Diagnosis and Prevention design pattern next because it focuses on a single aspect of a common misconception and the feedback targets a specific case. It is easier to create feedback for a single case rather than general feedback that considers multiple cases.

Finally, we felt that the Worked Examples design pattern required the most effort and is the reason why fewer preservice teachers selected it. Assuming that a teacher properly discussed a lesson, there would be fewer cases of students with very little understanding about the problem. The design pattern required preservice teachers to think about the steps in the solution process and explain each step clearly to ensure students could follow it. It might also be possible that some preservice teachers were not confident about their own understanding of the topic to construct effective examples.

Future work can investigate preservice teachers' reasoning behind their choice of design patterns and provide specific learning scenarios to check whether they are capable of selecting and applying appropriate design patterns.

Some preservice teachers' feedback messages were unclear, possibly due to their confusion with mathematical concepts. Design patterns that focus on structure or presentation may be ineffective when one's mathematical content knowledge is faulty. It is imperative that preservice teachers are not only taught to use design patterns, but also possess a sound knowledge of the mathematical concepts that they will be teaching.

The creation of video feedback in Task 3 was more complex compared to Tasks 1 and 2 because it considered additional aspects for feedback delivery. The change in the feedback delivery medium (i.e., video) reveals more aspects that need to be considered for this type of feedback. For example, some preservice teachers used illustrations to explain the Venn diagram which seemed more effective compared to spoken descriptions.

Preservice teachers need four skills to use educational design patterns for feedback effectively. First, identify the design pattern that will appropriately address the issues in a given learning situation. Second, identify math content that is relevant to the topic at hand. Third, construct feedback that follows the design pattern and utilizes appropriate mathematical content. Finally, deliver the feedback using a specific medium in a learning environment.

\section{SUMMARY AND FUTURE WORK}

Educational design patterns encapsulate knowledge from teaching experience and research. They are easily shared and can be applied by novice teachers to leverage existing knowledge instead of repeating the process of finding effective strategies that other teachers and researchers have already done.

Educational design patterns promote effective pedagogies because they are structured clearly and address specific aspects of student learning. They can reduce the cognitive load required to learn pedagogies by focusing on specific aspects of the strategy. The patterns used in this study, for example, helped preservice teachers focus on the learning context and feedback strategies instead of considering different aspects that come into play in a learning scenario.

This study investigated the potential of using educational design patterns to transfer pedagogical knowledge in math preservice teacher education. The results were promising as it showed that preservice teachers understood our selected design patterns, namely Feedback Sandwich, Pitfall Diagnosis and Prevention, and Worked Examples, and successfully applied the patterns after only receiving a short lecture about them. Preservice teachers used knowledge from the design patterns to construct feedback that addressed important aspects of students' learning experience such as students' self-regard, misconceptions, and prior knowledge. These characteristics were not common in naturalistic feedback they constructed before getting introduced to design patterns.

The study's results revealed that the selected design patterns addressed only part of the issues required for giving feedback. We do not have clear evidence to explain why preservice teachers selected certain design patterns and it should be explored in future work. Evaluating options for choosing a design pattern for a particular problem and a particular student adds a layer of complexity to the remedial feedback that teachers give. There are several other factors that need to be considered based on the context of the learning experience. In the case of constructing video feedback for a computer-based learning system, for example, the design may consider video duration, teaching materials used, frequency, and redundancy of feedback. Video feedback has many similarities with face-to-face feedback so the patterns used in this context can potentially be used in face-to-face settings as well [10].

The study was conducted on a single class of 32 preservice teachers in one university. A more rigorous study should be conducted to get accurate measures of design patterns' effectiveness as a teaching tool for math preservice teacher education in other institutions and student populations. Furthermore, the study may be replicated in other domains like English, Chemistry, Biology, and Computer Science to investigate the generalizability of design patterns as a teaching tool.

In future work, preservice teachers may need to be introduced to a design pattern language rather than a limited set of design patterns. Pattern languages describe interrelationships of different design patterns that can help design feedback that address multiple problems simultaneously. For example, incorporating design patterns that consider the duration of an explanation and resources needed for effective video feedback. Such research will most likely lead to more questions such as: What sequence should be followed 
Paul Salvador Inventado, Sharris Gayle Francisco Inventado, Noboru Matsuda, Yeping Li, Peter Scupelli, Korinn Ostrow, Neil Heffernan, EuroPLoP '18, July 4-8, 2018, Irsee, Germany Shihfen Tu, Craig Mason, Mary Logue, and Pat McGuire

in teaching design patterns? How should preservice teachers be taught to match design patterns to remediating student errors? What would be the scope of a course that utilizes a design pattern language? How should such a course be evaluated?

This study only considers the utility of design patterns for teacher preparation, but we also need to investigate how this translates to student learning. In order to see the real value of design patterns, we need to check how well preservice teachers retain pedagogical knowledge, whether they are able to apply this knowledge in real classrooms, and if this knowledge promotes better student learning. Future studies can compare student performance when they are taught by novice teachers who learned design patterns and novice teachers who did not.

Design patterns have a huge potential to be used as effective tools to improve preservice teacher education. Further studies can help investigate how we might properly leverage design patterns to support teachers and improve student success.

\section{ACKNOWLEDGEMENTS}

We would like to thank our shepherd Elisaveta Gurova and all participants in the writing workshop who helped us refine this paper. This material is based upon work supported by the National Science Foundation under DRL-1252297.

\section{REFERENCES}

[1] Christopher Alexander, Sara Ishikawa, Murray Silverstein, Max Jacobson, Ingrid Fiksdahl-King, and Shlomo Angel. 1977. A pattern language: Towns, buildings, construction. Oxford University Press, Oxford, UK.

[2] Dana Anthony. 1996. Patterns for classroom education. In Pattern Languages of Program Design 2, James Coplien John Vlissides and Norman Kerth (Eds.) Addison-Wesley Longman Publishing Co., Inc., Boston, MA, 391-406.

[3] Joseph Bergin, Jutta Eckstein, Markus Volter, Marianna Sipos, Eugene Wallingford, Klaus Marquardt, Jane Chandler, Helen Sharp, and Mary Lynn Manns. 2012. Pedagogical patterns: advice for educators. Joseph Bergin Software Tools, New Paltz, NY.

[4] Eric Chung, Jason Hong, James Lin, Madhu Prabaker, James Landay, and Alan Liu 2004. Development and Evaluation of Emerging Design Patterns for Ubiquitous Computing. In Proceedings of the 5th Conference on Designing Interactive Systems: Processes, Practices, Methods, and Techniques (DIS '04). ACM, New York, NY, USA 233-242.

[5] Erich Gamma, Richard Helm, Ralph Johnson, and John Vlissides. 1995. Design Patterns: Elements of Reusable Object-oriented Software. Addison-Wesley Longman Publishing Co., Inc., Boston, MA, USA.

[6] Sigrun Gudmundsdottir and Lee Shulman. 1987. Pedagogical content knowledge in social studies. Scandinavian fournal of Educationl Research 31, 2 (1987), 59-70.

[7] Neil Heffernan and Cristina Heffernan. 2014. The ASSISTments Ecosystem: Building a platform that brings scientists and teachers together for minimally invasive research on human learning and teaching. International fournal of Artificial Intelligence in Education 24, 4 (2014), 470-497.

[8] Takashi Iba. 2010. Learning Patterns: a pattern language for creative learning. CreativeShift Lab, Japan.

[9] Paul Salvador Inventado and Peter Scupelli. 2015. A Data-driven Methodology for Producing Producing Online Learning System Design Patterns. In Proceedings of the 22nd Conference on Pattern Languages of Programs (PLoP '15). The Hillside Group, USA, Article 26, 17 pages.

[10] Paul Salvador Inventado and Peter Scupelli. 2017. Adapting Design Patterns Across Learning Environments. In Proceedings of the VikingPLoP 2017 Conference on Pattern Languages of Program (VikingPLoP). ACM, New York, NY, USA, Article 10, 12 pages.

[11] Paul Salvador Inventado, Peter Scupelli, Eric Van Inwegen, Korinn Ostrow, Neil Heffernan III, Jaclyn Ocumpaugh, Ryan Baker, Stefan Slater, and Victoria Almeda. 2016. Hint Availability Slows Completion Times in Summer Work.. In Proceedings of the 9th International Conference on Educational Data Mining, Min Chi Tiffany Barnes and Mingyu Feng (Eds.). 388-393.

[12] Christian Kohls. 2012. Patterns for Creative Thinking. In Proceedings of the 19th Conference on Pattern Languages of Programs (PLoP '12). The Hillside Group, USA Article 3, 14 pages.
[13] Christian Köppe, Ralph Niels, René Bakker, and Stijn Hoppenbrouwers. 2016. Flipped Classroom Patterns: Controlling the Pace. In Proceedings of the 10th Travelling Conference on Pattern Languages of Programs (VikingPLoP '16). ACM, New York, NY, USA, Article 1, 13 pages.

[14] Mary Manns and Linda Rising. 2004. Fearless Change Patterns for Introducing New Ideas (first ed.). Addison-Wesley Professional, Boston, MA, USA.

[15] Yishay Mor. 2011. Embedding design patterns in a methodology for a design science of e-Learning. In Investigations of E-Learning Patterns: Context Factors, Problems and Solutions. IGI Global, PA, 107-134.

[16] Yishay Mor, Steven Warburton, and Niall Winters. 2012. Participatory pattern workshops: a methodology for open learning design inquiry. Research in Learning Technology 20 (2012).

[17] Jenifer Tidwell. 2010. Designing interfaces: Patterns for effective interaction design. O'Reilly Media, Inc., Sebastopol, CA, USA.

[18] Barbara Unger and Walter Tichy. 2000. Do design patterns improve communication? An experiment with pair design. In Proceedings of International Workshop Empirical Studies of Software Maintenance. 1-5.

[19] Steven Warburton and Yishay Mor. 2015. A set of patterns for the structured design of MOOCs. Open Learning: The fournal of Open, Distance and e-Learning 30, 3 (2015), 206-220.

[20] Marc Zimmermann, Daniel Herding, and Christine Bescherer. 2014. Pattern: Hint on Demand. In Practical Design Patterns for Teaching and Learning with Technology. SensePublishers, Netherlands. 